\title{
Maintenance of the Amygdala-Hippocampal Circuit Function with Safe and Feasible Shaking Exercise Therapy in SAMP-10 Mice
}

\author{
Runhong Yao Kazuhiro Nishii Naoki Aizu Takumi Kito Kazuyoshi Sakai \\ Kouji Yamada
}

Graduate School of Health Sciences, Fujita Health University, Toyoake, Japan

\section{Keywords}

Shaking exercise $\cdot$ Amygdala $\cdot$ Hippocampus · Passive avoidance task · Dementia

\begin{abstract}
Introduction: Patients with dementia show reduced adaptive, behavioral, and physiological responses to environmental threats. Physical exercise is expected to delay brain aging, maintain cognitive function and, consequently, help dementia patients face threats and protect themselves skillfully. Methods: To confirm this, we aimed to investigate the effects of the shaking exercise on the avoidance function in the senescence-accelerated mouse-prone strain-10 (SAMP10) model at the behavioral and tissue levels. SAMP-10 mice were randomized into 2 groups: a control group and a shaking group. The avoidance response (latency) of the mice was evaluated using a passive avoidance task. The degree of amygdala and hippocampal aging was evaluated based on the brain morphology. Subsequently, the association between avoidance response and the degree of amygdala-hippocampal aging was evaluated. Results: Regarding the passive avoidance task, the shaking group showed a longer latency period than the control group $(p<0.05)$, even and low intensity staining of ubiquitinated protein, and had a higher number of and larger neurons than those of the control
\end{abstract}

karger@karger.com www.karger.com/dee

Karger $\stackrel{\text { ' }}{5}$
(C) 2021 The Author(s)

Published by S. Karger AG, Basel

This is an Open Access article licensed under the Creative Commons Attribution-NonCommercial-4.0 International License (CC BY-NC) (http://www.karger.com/Services/OpenAccessLicense), applicable to the online version of the article only. Usage and distribution for commercial purposes requires written permission. group. The difference between the groups was more significant in the $B A$ region of the amygdala and the $C A 1$ region of the hippocampus (staining degree: $p<0.05$, neuron size: $p<$ 0.01 , neuron counts: $p<0.01$ ) than in other regions. Conclusions: The shaking exercise prevents nonfunctional protein (NFP) accumulation, neuron atrophy, and neuron loss; delays the aging of the amygdala and hippocampus; and maintains the function of the amygdala-hippocampal circuit. It thus enhances emotional processing and cognition functions, the memory of threats, the skillful confrontation of threats, and proper self-protection from danger.

(c) 2021 The Author(s)

Published by S. Karger AG, Basel

\section{Introduction}

Elderly individuals show reduced neurophysiological activity patterns, sensitivity to threat perception, and reflex recognition when compared to younger individuals [1]. The rate of exposure of patients with dementia to daily-life threats, such as falling [2] and traffic accidents [3] is high. These facts are very worrying. There is an urgent need to improve the sensitivity to threat perception and reflex recognition among elderly individuals to help them protect themselves.
Correspondence to:

Kouji Yamada, yamada@fujita-hu.ac.jp 
It is well known that the ability of animals to be aware of a threat, take measures to avoid it, and protect themselves is aided by disparate neural systems, which act independently and interactively to influence behavioral responses [4]. This involves a series of processes, such as fear generation, contextual learning, emotional memory, and defense response, which are mainly completed by the amygdala-hippocampal circuit [4]. The neuronal activity of the amygdala is very important for the prediction and perception of harmful threats [5]. However, the activation of the amygdala decreases with the advancement of age [6]. The amygdala frequently undergoes significant degeneration early in the course of Alzheimer's disease (AD) [6]. In addition, patients with AD display alterations in emotional expression [7]. Furthermore, functional brain imaging studies have confirmed the involvement of the hippocampus in both the formation (encoding) of new episodic memories and their subsequent retrieval [8]. It is considered to be the most age-sensitive system, with an average age of decline onset of approximately 60 years [8]. A previous study showed that fear conditioning gradually decays from healthy elderly individuals to patients with $\mathrm{AD}$, with patients with amnestic mild cognitive impairment (aMCI) showing an intermediate level decay [9]. However, methods to overcome this age-related defect remain unknown. We are devoted to exploring effective methods for overcoming age-related defects, increasing sensitivity to threat perception, and recognizing the reflexes in elderly individuals, to enable them to maintain their self-protection ability and reduce the associated social burden.

Physical exercise sensitizes the nervous system by promoting many processes, e.g., synaptic plasticity, neurogenesis, angiogenesis, and autophagy, as well as protective and preventive activities, e.g., improvements in memory, cognition, and mood [10]. Furthermore, 10 weeks of treadmill training was found to increase the dendritic arbor of the amygdala and hippocampus and enhance amygdala- and hippocampus-associated neuronal function in mice [11]. Taking into account the physiological characteristics of elderly individuals, a laboratory-designed shaking exercise has been developed and shown to be a safe and convenient method of physical exercise [1214]. The shaking exercise focuses on increasing physical activity by increasing muscle contraction. It is applied by using a rotating device with a horizontal panel for the subject to stand on. When the subject stands on a rocking surface, the rocking affects their balance. To avoid falling, the body will unconsciously reflex to maintain its balance, and the muscles begin to contract. When the shaking ex-

Shaking Exercise for Antiaging of

Amygdala-Hippocampal Circuit Function ercise is applied in a mouse model, we can ensure that all mice in the experiment are exposed to the same external force from a $360^{\circ}$ stimulation, thereby ensuring the objectivity of the experiment. Through experiments on mice, previous studies have proven that the shaking exercise can prevent a decline in lumbar [12] and thigh-bone mineral density [14], delaying the onset of arthritis [13] and slowing its progress.

A previous study provided preliminary evidence suggesting that the shaking exercise protects the learning and memory function of the hippocampus [15]. However, none of the previous studies have explored the effect of the shaking exercise on the amygdala-hippocampal circuit.

Based on the findings of previous studies, we hypothesized that the shaking exercise would delay the aging of the amygdala and hippocampus and help to maintain the function of the amygdala-hippocampal circuit, thereby maintaining the emotional processing function, cognition function, and the memory of threats, enhancing skillful confrontation of threats and enabling proper selfprotection from danger.

\section{Materials and Methods}

\section{Animals}

Eight-week-old male SAMP-10 mice (SAMP10/TaSlc, SLC, Shizuoka) were used. The mice were randomly assigned to 2 groups (shaking group, $n=12$; control group, $n=12$ ). The timing of the passive avoidance task and sample collection was set based on senescence and health.

\section{Shaking Exercise}

Twelve mice were placed in 12 separate spaces on the shaking machine. During the exercise, they remained isolated and did not interfere with each other. Shaking stimulation involved a horizontal rotation movement with a variable axis of rotation that was uniformly applied in all directions (NR-3; TAITEC Co. Ltd., Saitama). Stimulation parameters were: movement distance, $50 \mathrm{~mm}$; shaking speed, 150 times/min. The stimulation was applied for 30 min/day 3 times/week for 25 continuous weeks. The intervention was initiated when the mice were aged 9 weeks and terminated when they were aged 33 weeks (Fig. 1).

\section{Passive Avoidance Task}

According to the results of senescence and health, the passive avoidance task (STC-001M and SGS-003DX, Muromachi Kikai Co. Ltd., Tokyo) was carried out at the age of 33 weeks. On the first day (memory acquisition), each mouse was placed in an illuminated space. After $1 \mathrm{~min}$, a sliding door was opened, and the mouse was given access via a narrow hole into a closed dark compartment. After the mouse had encountered the open door for the first time, their latency to enter the dark compartment was measured. When all 4 paws of the mouse had entered the dark room, the door was 


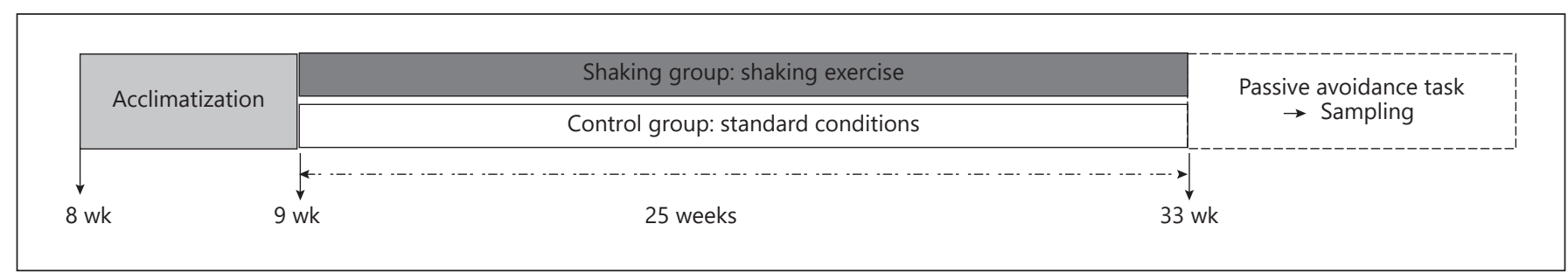

Fig. 1. Shaking exercise schedule. Before the shaking exercise, the shaking group was habituated for 1 week, and the shaking stimulation was started when the mice were 9 weeks (wk) old. The shaking exercise lasted for 25 weeks. We conducted the passive avoidance task analysis and sampling when the mice were 33 weeks old.

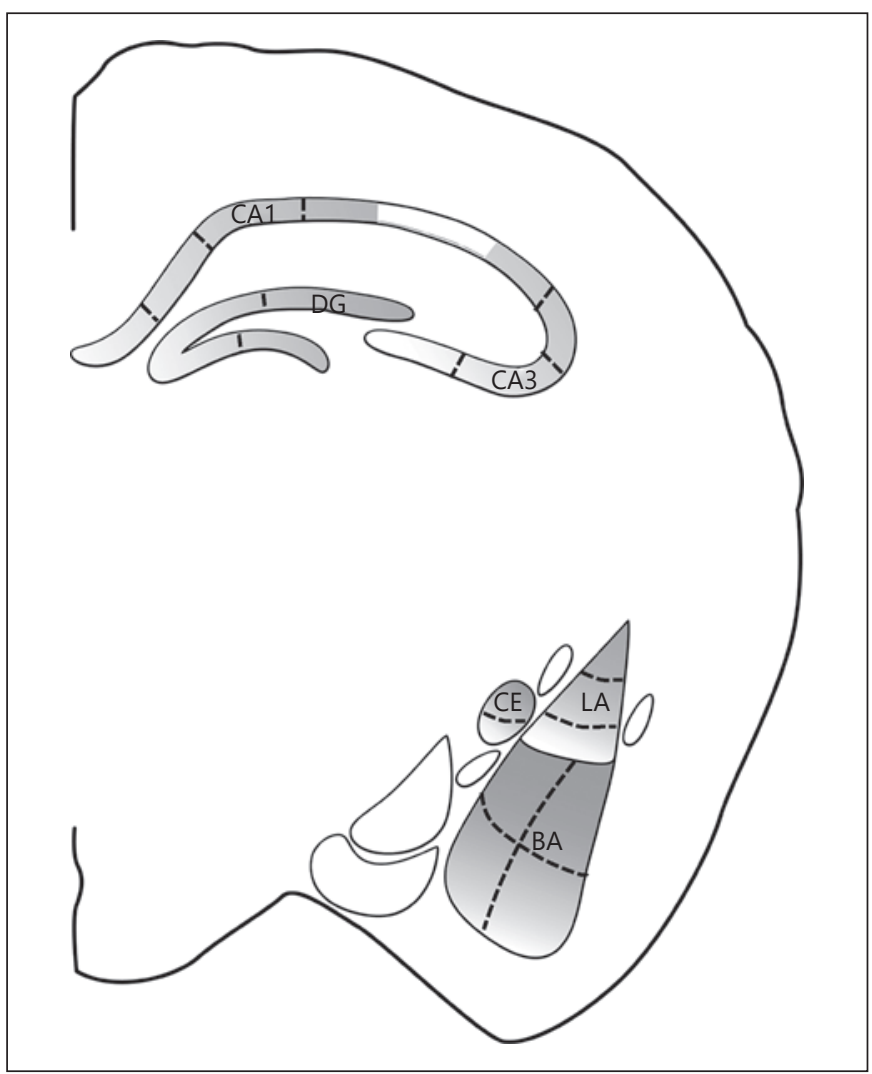

Fig. 2. The amygdala was divided into the lateral amygdala (LA), basal amygdala (BA), and central nucleus (CE). The hippocampus was divided into the dentate gyrus (DG), cornu ammonis field 3 (CA3), and cornu ammonis field 1 (CA1). The degree of ubiquitinpositive staining, the neuron size, and the number of neurons were measured using 6 different regions of each tissue block.

closed, and a foot shock ( $3 \mathrm{~s}, 0.25 \mathrm{~mA}$ ) was delivered. The next day (memory regeneration), each mouse was again placed in the illuminated room. The latency before entering the darkroom (up to 300 s) was measured.

\section{Histomorphology Analysis}

Brain samples were immediately stored in formalin and embedded in paraffin wax. Coronal sections $(20-\mu \mathrm{m}$-thick) of brain slices containing the central zone of the hippocampus (approx. 3 $\mathrm{mm}$ posterior to the bregma) were made using a microtome. These sections were immunostained according to the standard method.

The amygdala was divided into 3 parts (lateral amygdala [LA], basal amygdala [BA], and central nucleus [CE]) and the hippocampus was divided into 3 parts (dentate gyrus [DG], cornu ammonis field 3 [CA3], and cornu ammonis field 1 [CA1]) (Fig. 2). The intensity of ubiquitin-positive staining, the neuron size, and the neuron count in all 6 regions was measured using ImageJ software.

\section{Statistical Analysis}

SPSS v24.0 (SPSS, Chicago, IL, USA) was used. $p<0.05$ was considered significant. Student's $t$ test was used to compare the results of the passive avoidance task and the histomorphometry analyses between the groups.

\section{Results}

During the passive avoidance task, on the first day (memory acquisition), there were no differences in latency between the 2 groups $(24.50 \pm 6.43$ vs. $23.08 \pm 3.20 \mathrm{~s}$, $t[16.14]=0.68$, ns). However, on the next day (memory regeneration), the shaking group showed a significantly longer latency $(249.09 \pm 57.73$ vs. $150.90 \pm 90.91 \mathrm{~s}$, $t[14.9]=2.92, p<0.05)$ (Fig. 3).

With regard to histomorphology, the shaking group showed even and low-intensity staining, which indicated low ubiquitinated protein accumulation. Additionally, the shaking group had larger neurons and a higher number of neurons. Neuronal loss was higher in the control group than in the shaking group, especially in the BA and CA1 regions (Fig. 4, 5).

The amygdala and hippocampus are shown in the whole image of the immunostained photomicrographs (Fig. 4a, e). The levels of ubiquitinated proteins were analyzed according to the intensity of the staining. 


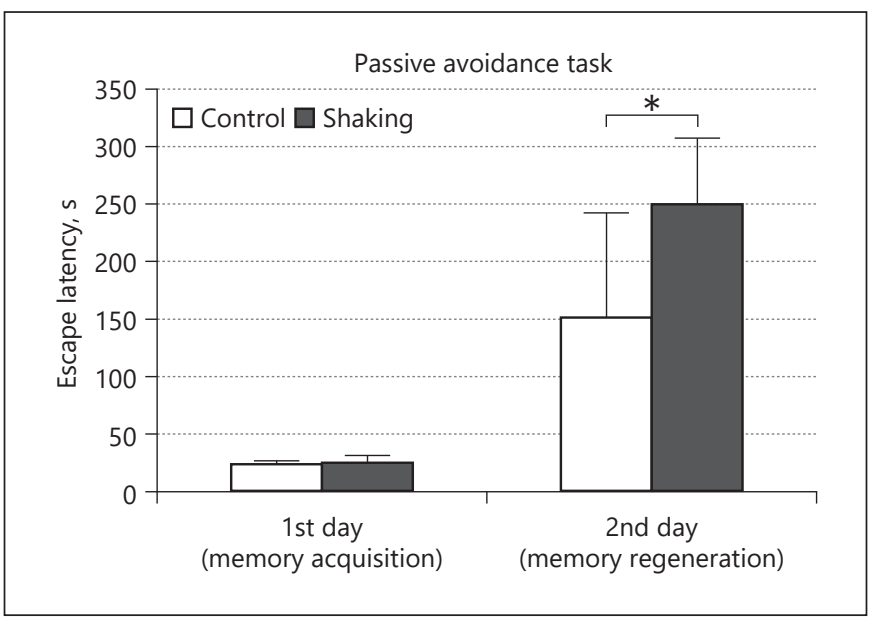

Fig. 3. The graph shows the results of the passive avoidance task. The tests were conducted at the age of 33 weeks. The latency before entering the darkroom was measured. Fear data were acquired the first time. The related memory regeneration after $24 \mathrm{~h}$ was measured. ${ }^{*} p<0.05$.

The degree of staining in the shaking group was significantly lower than that in the control group in the LA $(163.77 \pm 5.26$ vs. $154.90 \pm 5.70, t[10]=2.80, p<0.05), \mathrm{BA}$ $(160.99 \pm 7.74$ vs. $144.52 \pm 11.07, t[10]=2.99, p<0.05)$, $\mathrm{CE}(163.43 \pm 6.54$ vs. $153.49 \pm 7.77, t[10]=2.40, p<0.05)$, and CA1 $(169.07 \pm 1.49$ vs. $160.26 \pm 7.72, t[5.37]=2.75$, $p<0.05$; Fig. 4b-d, h; 5a). There were no differences between the groups in the degree of staining in the DG $(166.35 \pm 3.80$ vs. $160.58 \pm 6.61, t[10]=1.85, \mathrm{~ns})$ or CA3 $(159.57 \pm 6.33$ vs. $158.21 \pm 7.71, t[10]=0.33$, ns) (Fig. $4 \mathrm{f}, \mathrm{g} ; 5 \mathrm{a})$.

The neuronal area was significantly larger in the shaking group than in the control group in the BA $(80.94 \pm$ 5.45 vs. $\left.65.36 \pm 9.75 \mu \mathrm{m}^{2}, t[10]=3.41, p<0.01\right), \mathrm{CE}$ $\left(73.48 \pm 8.09\right.$ vs. $\left.53.91 \pm 7.51 \mu \mathrm{m}^{2}, t[10]=4.34, p<0.01\right)$, DG $\left(53.73 \pm 2.65\right.$ vs. $38.97 \pm 5.69 \mu \mathrm{m}^{2}, t[7.08]=5.76, p<$ $0.01), C A 3\left(92.84 \pm 6.13\right.$ vs. $81.16 \pm 7.93 \mu \mathrm{m}^{2}, t[10]=2.85$, $p<0.05)$, and CA1 $\left(88.37 \pm 6.02\right.$ vs. $61.31 \pm 3.52 \mu \mathrm{m}^{2}$, $t[10]=2.75, p<0.01$; Fig. $4 c, d, f-h ; 5 b)$. There were no significant differences between the groups in the LA $(66.75 \pm 4.47$ vs. $64.34 \pm 5.65, t[10]=0.82$, ns; Fig. $4 b ; 5 b)$.

The number of neurons was significantly higher in the shaking group than in the control group in the BA $(41.75 \pm 4.28$ vs. $16.75 \pm 6.63, t[10]=7.76, p<0.01), \mathrm{CE}$ $(50.17 \pm 9.44$ vs. $33.92 \pm 8.17, t[10]=3.19, p<0.05), \mathrm{DG}$ $(157.89 \pm 23.1$ vs. $112.50 \pm 26.71, t[10]=3.15, p<0.05)$, CA3 (46.54 \pm 4.14 vs. $39.08 \pm 6.15, t[10]=2.47, p<0.05)$, and CA1 $(67.08 \pm 4.7$ vs. $39.42 \pm 11.31, t[10]=5.53, p<$
0.01; Fig. $4 c, d, f-h ; 5 c)$. There were no significant differences between the groups in the number of neurons in the LA $(46.78 \pm 4.53$ vs. $40.72 \pm 5.76, t[10]=2.02$, ns; Fig. 4 b; $5 c)$.

\section{Discussion}

The amygdala is essential for emotional association and response, while it has been suggested that the hippocampus is involved in the unconditioned stimulus-conditioned stimulus (US-CS) learning of both the factual information regarding the stimuli and the links between contextual cues [16]. The interaction of these 2 regions transforms fear into darkroom avoidance behavior.

SAMP-10 mice in the shaking group showed a long latency period before entering the darkroom, which proved that the mice had maintained their avoidance function and protected themselves from the threat of foot shock after the shaking exercise. With regard to the avoidance behavior, mice are believed to experience 2 forms of fear. The first is the learning of fear, which involves exposure to the real threat caused by the foot shock threat when entering the darkroom. The second is the regeneration of fear, which is caused by brain association. The mice connected the learned darkroom contextual CS to the foot shock US when they hovered at the threshold. The shaking group showed low-intensity ubiquitin-positive staining in the BA and CE regions as well as a high number of and large neurons. In the shaking group, fear was processed in the BA area through healthy neurons and the corresponding signal was transmitted completely and smoothly through the CE; it entered the hippocampus through the olfactory cortex [17]. In the DG-CA3CA1 trisynaptic pathway of the hippocampus, the DG received the contextual information of the darkroom and the fear of the foot shock from the amygdala [18]. The information was processed rapidly in the autoassociative CA3 network, sent to the CA1, and then stored [19]. At this moment, the hippocampus completed the learning and creation of the memory of entering the darkroom.

In addition, the nonfunctional protein (NFP) deposition level was low in the CA1 region of the shaking group. We can imagine that the hippocampal neurons in the relatively healthy shaking group obtained a more reliable memory of the darkroom context and fear of the foot shock than did the control group. After $24 \mathrm{~h}$, when the mice were close to the dangerous border, i.e., the entrance to the darkroom, the shaking group lingered in the light room for a longer time and showed the expected avoid- 
Fig. 4. Histomorphology structure of the partial amygdala and hippocampus. a, e Photomicrographs show the whole image of the anti-ubiquitin antibody-immunostained amygdala and hippocampus. Scale bars, $200 \mu \mathrm{m}$. b-d LA, BA, CE of the amygdala. $\mathbf{f}-\mathbf{h}$ DG, CA3, and CA1 of the hippocampus. Scale bars, $50 \mu \mathrm{m}$.

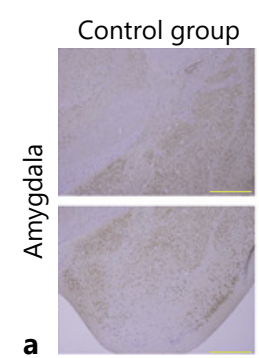

a

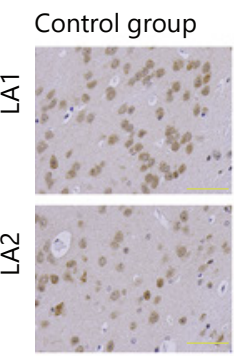

$\stackrel{m}{\leftrightarrows}$

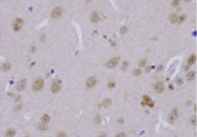

b
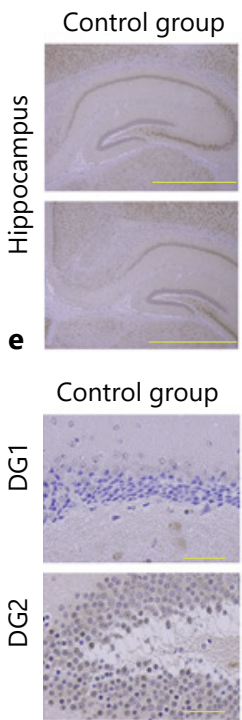

ญ̆

m

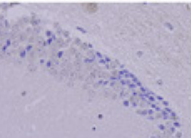

f

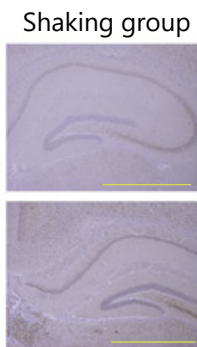

Shaking group
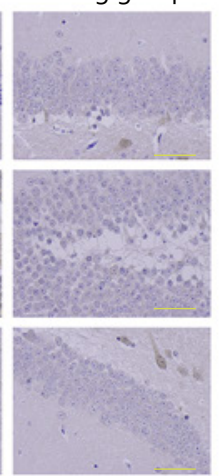

Shaking group

这

Control group
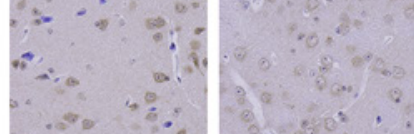

$\underset{\infty}{\mathfrak{1}}$
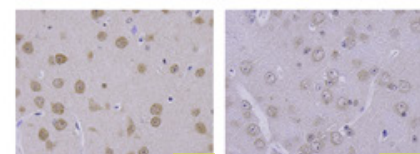

\ุ
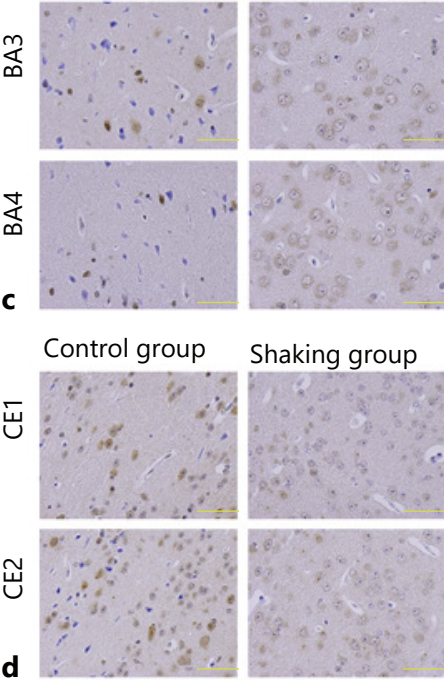

Shaking group
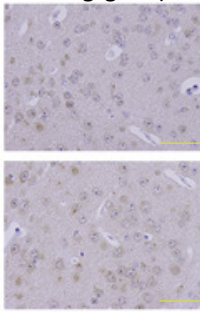

Control group

Shaking group
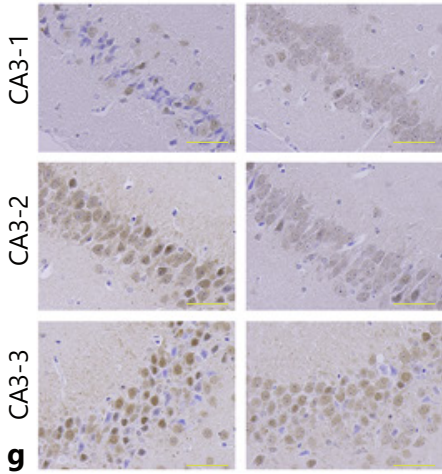

Control group

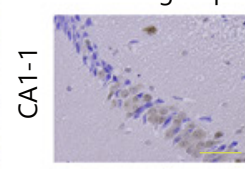

Shaking group
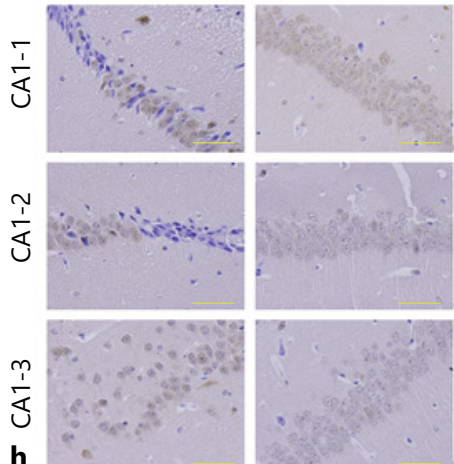

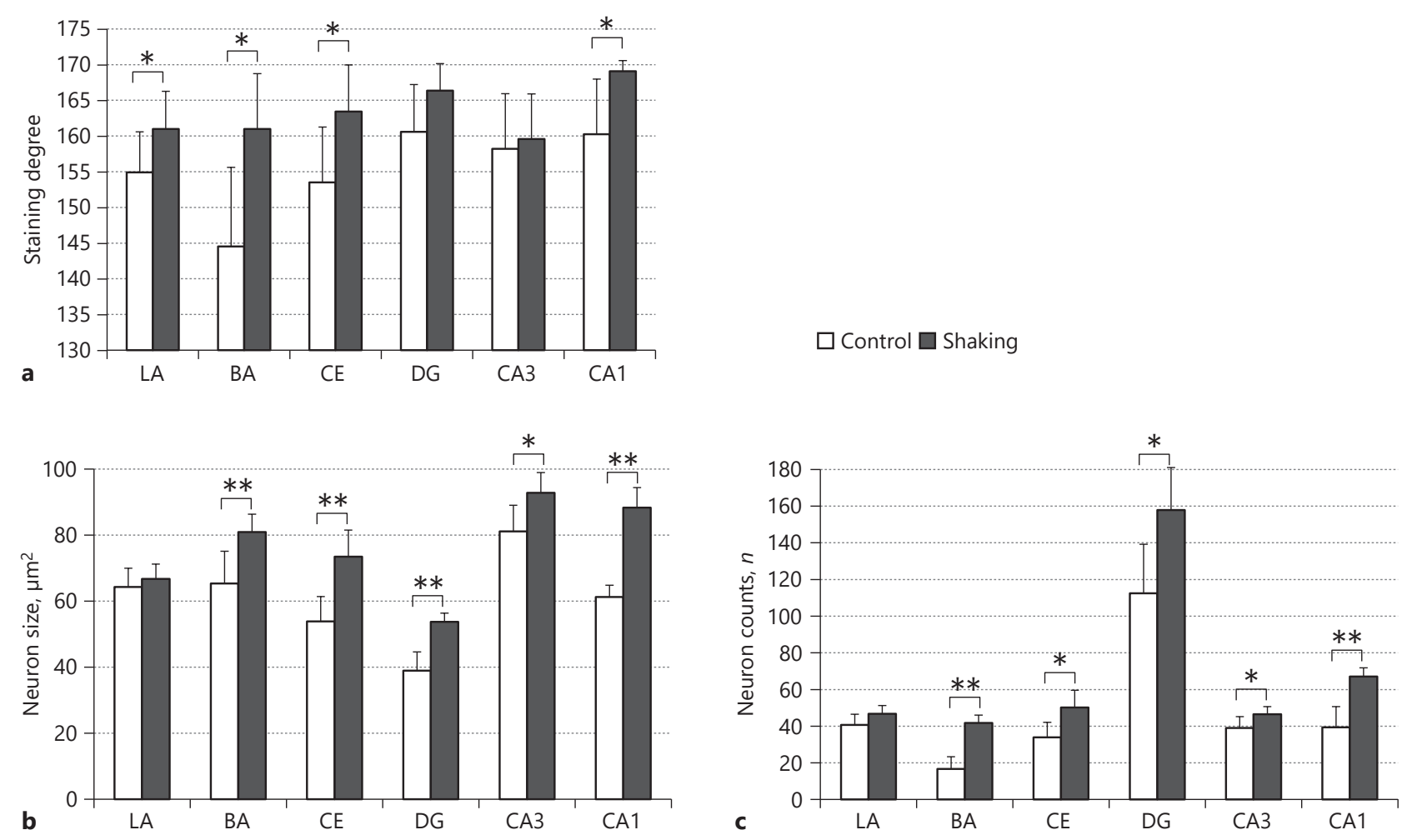

Fig. 5. Histomorphology analysis of the amygdala and hippocampus. a The degree of ubiquitin-positive staining in the amygdala and hippocampus. $\mathbf{b}$ The neuron size in the amygdala and hippocampus. $\mathbf{c}$ The number of neurons in the amygdala and hippocampus. ${ }^{*} p<0.05,{ }^{* *} p<0.01$.

ance response. The darkroom context as a retrieval cue, when the mice return to the familiar context, led to the activation of the relevant memory. The hippocampal neurons of the shaking group maintained the ensemble function of each region of the hippocampus, which made it easier to retrieve the right responses to the environment.

Specifically, the DG also plays a key role in the disambiguation of memory. The DG in the shaking group easily eliminated unnecessary memory interference [20], thereby helping the hippocampal CA3 and CA1 regions to support the fear memory and recognize the darkroom context. The key to the avoidance reaction is the coupling of the CS-US. CS-US processing takes place in the CA1 region. In addition, the CA1 region helps recognition of the novelty or familiarity of the object or context [19]. In the shaking group, the intact CA1 managed the combination of darkroom memory and fear perfectly. At this stage, the shaking group successfully completed the ensemble of the DG-CA3-CA1 region in the hippocampus and became a coherent system which carried the necessary information of the avoidance reaction, linked the memory with the right context, and provided a mechanism to trigger an appropriate avoidance response [21]. The regulation of emotional arousal on the consolidation of avoidance response occurs in the BA [22]. After the BA received information from the hippocampus, fear was again aroused in the BA. Because the shaking group had more healthy neurons, it could activate the BA more smoothly than the control group could, to arouse fear and lay the foundation for a fear-avoidance response. As a result, fear flowed through the amygdala to the hypothalamus, causing fear responses controlled by the central nucleus [23].

In this study, in contrast to the difference in the accumulation of NFP and the neuron counts, the difference in 
size between the 2 groups was more significant (Fig. 4, 5). We infer that the aging of the amygdala and hippocampus in 33-week-old SAMP mice was mainly manifested by the accumulation of NFP and the gradual atrophy of neurons, but not by the initiation of complete neuron loss. By means of the shaking exercise, the accumulation of NFP was reduced and progression of neuronal senescence into the neuron atrophy stage was prevented. In addition, in the 2 circuits of avoidance behavior, the BA and CA1 play a more decisive role. The health of the BA and CA1 in the shaking group was more significant than in the control group (Fig. 5). This could indicate that the BA and CA1 are more easily affected by shaking exercise.

It has been reported that, after 6 months of physical exercise, the volume of the hippocampus of patients with aMCI increased and their performance in the episodic memory test improved [24]. Exercise can affect hippocampal growth, synaptic plasticity, and cue-extraction induced by exercise [25]. These studies support our results that physical exercise has a positive impact on amygdalahippocampal activation and emotional memory processing.

We believe that the shaking exercise can delay the aging of the amygdala and hippocampus; maintain the function of the amygdala-hippocampal circuit to promote the maintenance of emotional sensitivity; improve the cognition of potential threats; enhance skillful avoidance behavior; and improve proper self-protection from danger. We recommend that elderly individuals maintain a healthy lifestyle through physical exercise, to maintain their emotional sensitivity and improve their awareness of potential risks, and thus protect themselves from danger.

\section{Acknowledgement}

This work was supported by grants-in-aid for scientific research from the Japan Society for the Promotion of Science and the Fujita Health University.

\section{Statement of Ethics}

All animals were maintained according to the protocols approved by the Institutional Animal Care and Use Committee of the Fujita Health University (approval No. H0702) and following the US National Institutes of Health (NIH) Guide for the Care and Use of Laboratory Animals. This article does not contain any data from studies on humans performed by any of the authors.

\section{Conflict of Interest Statement}

The authors have no conflicts of interest to declare.

\section{Funding Sources}

This work was supported by the Japan Society for the Promotion of Science (No. 22500484).

\section{Author Contributions}

R.Y. and K.Y. designed the project and wrote the manuscript and approved the final version to be published. R.Y. and K.N. performed the behavioral tests and participated in drafting the work. All the members took part in the anatomy experiment. K.Y., K.N., and T.K. performed the brain staining experiment. N.A. and K.S. performed the microscopy experiments and helped with the data analysis..

\section{References}

1 Houston JR, Pollock JW, Lien MC, Allen PA. Emotional arousal deficit or emotional regulation bias? An electrophysiological study of age-related differences in emotion perception. Exp Aging Res. 2018 MayJun;44(3):187-205.

2 Dolatabadi E, Van Ooteghem K, Taati B, Iaboni A. Quantitative Mobility Assessment for Fall Risk Prediction in Dementia: A Systematic Review. Dement Geriatr Cogn Disord. 2018;45(5-6):353-67.

3 Byszewski A, Power B, Lee L, Rhee GG, Parson B, Molnar F. Driving and dementia: Workshop module on communicating cessation to drive. Can Geriatr J. 2017 Dec; 20(4):241-5.
4 Lindquist DH. Emotion in motion: A threestage model of aversive classical conditioning. Neurosci Biobehav Rev. 2020 Aug;115:36377.

5 Blair HT, Sotres-Bayon F, Moita MA, Ledoux JE. The lateral amygdala processes the value of conditioned and unconditioned aversive stimuli. Neuroscience. 2005;133(2):561-9.

6 Allen PA, Lien MC, Jardin E. Age-related emotional bias in processing two emotionally valenced tasks. Psychol Res. 2017 Jan;81(1):289-308.

7 Hamann S, Monarch ES, Goldstein FC. Impaired fear conditioning in Alzheimer's disease. Neuropsychologia. 2002;40(8):1187-95.

8 Nyberg L. Functional brain imaging of episodic memory decline in ageing. J Intern Med. 2017 Jan;281(1):65-74.
9 Nasrouei S, Rattel JA, Liedlgruber M, Marksteiner J, Wilhelm FH. Fear acquisition and extinction deficits in amnestic mild cognitive impairment and early Alzheimer's disease. Neurobiol Aging. 2020 Mar;87:26-34.

10 Mahalakshmi B, Maurya N, Lee SD, Bharath Kumar V. Possible neuroprotective mechanisms of physical exercise in neurodegeneration. Int J Mol Sci. 2020 Aug;21(16):E5895.

11 Lin TW, Shih YH, Chen SJ, Lien CH, Chang CY, Huang TY, et al. Running exercise delays neurodegeneration in amygdala and hippocampus of Alzheimer's disease (APP/PS1) transgenic mice. Neurobiol Learn Mem. 2015 Feb;118:189-97. 
12 Yamada K, Nishii K, Sakai K, Teranishi T. Stimulus in the form of rotation and shaking of a platform and its effect on the formation of trabecular bone in the lumbar vertebrae of mice. Aging Clin Exp Res.2013 Dec;25(6):62532.

13 Kito T, Teranishi T, Nishii K, Sakai K, Matsubara M, Yamada K. Effectiveness of exercise-induced cytokines in alleviating arthritis symptoms in arthritis model mice. Okajimas Folia Anat Jpn. 2016;93(3):81-8.

14 Yao R, Nishii K, Kito T, Teranishi T, Sugiyama T, Sakai K, et al. A novel device to prevent osteoporosis by promoting bone metabolism using a newly developed double-loading stimulation with vibration and shaking. Okajimas Folia Anat Jpn. 2019;96(1):13-21.

15 Yao R, Nishii K, Aizu N, Kito T, Sakai K, Yamada K. Maintaining Aging Hippocampal Function with Safe and Feasible Shaking Exercise in SAMP10 Mice. Dement Geriatr Cogn Disord. 2020;49(2):185-93.
16 Arias N, Méndez M, Arias JL. The importance of the context in the hippocampus and brain related areas throughout the performance of a fear conditioning task. Hippocampus. 2015 Nov;25(11):1242-9.

17 Fadok JP, Markovic M, Tovote P, Lüthi A. New perspectives on central amygdala function. Curr Opin Neurobiol. 2018 Apr;49:1417.

18 Rolls ET, Critchley HD, Treves A. Representation of olfactory information in the primate orbitofrontal cortex. J Neurophysiol. 1996 May;75(5):1982-96.

19 Daumas S, Halley H, Francés B, Lassalle JM. Encoding, consolidation, and retrieval of contextual memory: differential involvement of dorsal CA3 and CA1 hippocampal subregions.Learn Mem.2005Jul-Aug;12(4):375-82.

20 Gao A, Xia F, Guskjolen AJ, Ramsaran AI, Santoro A, Josselyn SA, et al. Elevation of hippocampal neurogenesis induces a temporally graded pattern of forgetting of contextual fear memories. J Neurosci. 2018 Mar;38(13):31908.
21 Smith DM, Bulkin DA. The form and function of hippocampal context representations. Neurosci Biobehav Rev. 2014 Mar;40:52-61.

22 Park S, Choi S. Initial conditioning and reconditioning recruit different populations of 'fear neurons' in the basal amygdala of rats. Biochem Biophys Res Commun. 2020 Apr;525(2):292-7.

23 Paré D, Quirk GJ, Ledoux JE. New vistas on amygdala networks in conditioned fear. J Neurophysiol. 2004 Jul;92(1):1-9.

24 Teixeira CV, Ribeiro de Rezende TJ, Weiler M, Magalhães TN, Carletti-Cassani AF, Silva TQ, et al. Cognitive and structural cerebral changes in amnestic mild cognitive impairment due to Alzheimer's disease after multicomponent training. Alzheimers Dement (N Y). 2018 Aug;4:473-80.

25 Loprinzi PD, Edwards MK, Frith E. Potential avenues for exercise to activate episodic memory-related pathways: a narrative review. Eur J Neurosci. 2017 Sep;46(5):2067-77. 